\title{
ShengMai-San Attenuates Cardiac Remodeling in Diabetic Rats by Inhibiting NOX-Mediated Oxidative Stress
}

This article was published in the following Dove Press journal: Diabetes, Metabolic Syndrome and Obesity: Targets and Therapy

\section{Yanting Lu \\ Shu Zhu \\ Xiaoyan Wang \\ Juhai Liu \\ Yingying Li \\ Wei Wang \\ Shijun Wang \\ Furong Wang}

College of TCM, Shandong University of Traditional Chinese Medicine, Jinan, 250355, People's Republic of China
Correspondence: Shijun Wang;

Furong Wang

Email wsj@sdutcm.edu.cn;

I5I68888786@I63.com
Background and Purpose: ShengMai-San (SMS) is traditionally used to treat ischemic cardiovascular and cerebrovascular diseases. Recently, several studies have reported the cardioprotective effects of SMS in diabetic animals. However, the potential mechanisms have not yet been fully elucidated. In this study, we investigated whether SMS exerts a beneficial effect in diabetic cardiomyopathy (DCM) by alleviating NADPH oxidase (NOX)-mediated oxidative stress.

Methods: SD rats were randomly divided into a negative control group (NC), diabetes mellitus group (DM) and SMS-treated group (SMS). The myocardial structure alterations, apoptosis and biomarkers of oxidative stress were observed. Moreover, to explore the protective mechanism of SMS, the activation of AMPK $\alpha$, expression and translocation of NOX-related proteins were assessed.

Results: Diabetes led to excessive collagen content, fibrosis, and apoptosis in the myocardium. Oxidative stress in diabetic hearts was indicated by low levels of T-AOC, high levels of 8-isoPGF2 $\alpha$ and 8-OHdG, inactivation of AMPK $\alpha$, elevated expression of NOX2 and NOX4 and translocation of NOX isoforms p47phox and p67phox. Treatment with SMS for 10 weeks resulted in the alleviation of diabetes-associated myocardial structure abnormalities and apoptosis. Additionally, SMS attenuated the accumulation of oxidative stress markers in myocardial tissue. Further investigation showed that SMS was able to reverse the levels of oxidative stress-associated proteins NOX2 and NOX4 in the DM rats. Moreover, SMS treatment blunted the translocation of NADPH oxidase isoforms p47phox and p67phox as well. Furthermore, SMS promoted the activation of AMPK in the cardiac tissue of diabetic rats.

Conclusion: These findings indicate that SMS exhibits therapeutic properties against diabetic cardiomyopathy by attenuating myocardial oxidative damage via activation of AMPK $\alpha$ and inhibition of NOX signaling.

Keywords: diabetes, myocardium, ShengMai-San, oxidative stress, AMPK, NOX

\section{Introduction}

Diabetes mellitus (DM) is the most widespread metabolic disease worldwide. It is estimated that approximately 347 million people suffer from diabetes globally. ${ }^{1}$ Diabetes is often accompanied by a wide spectrum of cardiovascular complications, which account for at least $80 \%$ of deaths among patients with diabetes. ${ }^{2}$ Independent of hypertensive, ischemic, or valvular heart diseases, diabetic cardiomyopathy (DCM) refers to a direct injury to the myocardium that is mediated by hyperglycemia and leads to both structural and functional abnormalities. ${ }^{3}$ The main 
characteristics of DCM are initial cardiac remodeling including left ventricular wall thickening, cardiomyocyte hypertrophy, apoptosis and interstitial fibrosis, followed by systolic and diastolic dysfunction, and ultimately heart failure and death.

Several mechanisms have been proposed to be involved in the pathogenesis of DCM. Under diabetic conditions, hyperglycemia, hyperlipidemia, insulin resistance, and other metabolic disorders lead to a series of abnormal cell functions, such as oxidative stress, mitochondrial dysfunction, production of advanced glycation end products (AGEs), impairment of calcium homeostasis, inflammation, activation of renin-angiotensin-aldosterone system (RAAS), and endoplasmic reticulum (ER) stress. ${ }^{4}$ Most of these abovementioned pathological and molecular alterations possess high redox sensitivity. Thus, oxidative stress is considered to be the most important inducer of DCM. Interventions targeting oxidative stress and the related signaling pathways may be a potential strategy for the prevention and treatment of DCM. ${ }^{5}$

ShengMai-San (SMS) is a traditional Chinese medicine formula that comprises Panax ginseng C. A. Mey. (sovereign), Ophiopogon japonicus (Thunb.) Ker Gawl. (minister), and Schisandra chinensis (Turcz.) Barll. (assistant). In China, SMS has been typically prescribed to prevent and treat cardiovascular diseases for more than 1000 years. At present, SMS is still used for the treatment of diabetes and various complications. Previous studies have shown that SMS improves cardiac function and delays the process of DCM by alleviating cardiomyocyte proliferation, myocardial hypertrophy, and interstitial fibrosis in type 2 diabetic rats and $\mathrm{db} /$ $\mathrm{db}$ mice. ${ }^{6-8}$ Using a systematic pharmacological methodology, Zhang et al found that SMS could regulate various signaling pathways and biological processes related to glucose and lipid metabolism, inflammation, and oxidative stress in the myocardium of diabetic rats, thus possessing a therapeutic value for DCM. ${ }^{9}$ However, the molecular mechanism of SMS in DCM remains unclear.

Therefore, in the present study, we investigated the effect and potential mechanism of SMS on a high-fat diet (HFD) combined with multiple low-dose injections of streptozotocin (STZ)-induced DCM. Our results indicated that SMS attenuates diabetes-induced alterations in the myocardial structure and apoptosis by activating adenosine monophosphate-activated protein kinase (AMPK), thereby inhibiting nicotinamide adenine dinucleotide phosphate oxidase (NOX) and the subsequent oxidative injuries.

\section{Materials and Methods Drugs and Reagents}

The decocting-free granules of Panax ginseng, Ophiopogon japonicus, and Schisandra chinensis were purchased from Tianjiang Pharmaceutical Co., Ltd (Jiangyin, Jiangsu, China). These three agents were mixed in a dry weight ratio of 1:3:1.5 and dissolved with distilled water before administration. Rabbit anti-NOX2, NOX4 and p67phox antibody were from Abcam (Cambridge, UK). Rabbit antiphospho-AMPK (Thr172) and AMPK antibody were purchased from Cell Signaling Technology (Boston, USA). Goat anti-p47phox antibody was from Sigma (St. Louis, MO, USA).

\section{Animals and Treatment}

All animal experiments were conducted as per the Guidelines of the National Institutes of Health (Bethesda, MD, USA) for the Care and Use of Laboratory Animals and approved by the Animal Care and Use Committee of Shandong University of Traditional Chinese Medicine (Jinan, China). Male Sprague-Dawley (SD) rats (aged 8 weeks with a body weight of 180-210 g) were obtained from Beijing Charles River Laboratory Animal Technology Co., Ltd. (Beijing, China). The animals were housed under a 12-h light/dark cycle with free access to food and water. The room temperature was maintained at $22 \pm 2{ }^{\circ} \mathrm{C}$ with a relative humidity of $55 \pm 5 \%$. After adaptation for 1 week, SD rats were fed an HFD for 4 weeks and then intraperitoneally injected with STZ (S0130, $25 \mathrm{mg} / \mathrm{kg}$; Sigma-Aldrich) prepared in 0.1 M sodium citrate buffer ( $\mathrm{pH}$ 4.5) 3 times every other week (Supplemental Figure 1). Three days after the last STZ injection, fasting blood glucose levels were measured using an Accu-CHEK active blood glucose meter (Roche, Mannheim, Germany). Animals with fasting blood glucose levels $\geq 11.1 \mathrm{mmol} / \mathrm{L}$ were considered to have diabetes and were randomly divided into two groups $(\mathrm{n}=15$ rats per group): a DM group and an SMS-treated group (SMS). DM and SMS rats were given a constant HFD and treated with normal saline or SMS $(1.8 \mathrm{~g} / \mathrm{kg} /$ day $)$ via intragastric administration. The negative control (NC) group received a normal diet and an intraperitoneal injection of citrate buffer and saline daily via gavage. After treatment with 
SMS or saline for consecutive 10 weeks, all rats were euthanized (Supplemental Figure 1). Ventricular tissues were collected and used for subsequent assays.

\section{Histopathological and Morphometric Analysis}

Heart tissues were collected from mice and washed with precooled saline, then fixed in $4 \%$ paraformaldehyde and embedded in paraffin. Five-millimeter slices of heart tissue were stained with haematoxylin eosin (HE) and Masson's trichrome staining. The morphological examination was performed using a light microscope (Olympus BX53, Japan). For transmission electron microscopy (TEM), left ventricle tissues were cut into pieces of $1 \times 1 \times 1 \mathrm{~mm}$ and fixed in $2.5 \%$ glutaraldehyde phosphate-buffered saline (PBS) and osmium tetraoxide (2\%). Tissues were then dehydrated and embedded in resin. Ultra-thin sections of $80 \mathrm{~nm}$ thickness were provided, and then placed in formvar-coated single-slot grids and counterstained with saturated aqueous uranyl acetate for $15 \mathrm{~min}$ and Reynolds lead citrate for $15 \mathrm{~min}$. Sections were examined using a HITACHI electron microscope (Hitachi, Japan).

\section{Terminal Deoxynucleotidyl Transferase-Mediated dUTP Nick End Labelling (TUNEL) Staining}

Apoptotic cardiomyocytes were visualized by TUNEL staining according to the manufacturer's instructions (KeyGEN Biotech, Nanjing, China). Briefly, myocardial tissues were fixed with $4 \%$ paraformaldehyde. Paraffin sections were dehydrated with graded alcohol, dewaxed in xylene and digested with proteinase $\mathrm{K}(10-20 \mu \mathrm{g} / \mathrm{mL})$ for $15 \mathrm{~min}$ at room temperature. Then, the sections were washed with PBS for three times (5 min each) and incubated with $50 \mu \mathrm{L}$ TUNEL reaction mixture in a $37^{\circ} \mathrm{C}$ dark chamber for $60 \mathrm{~min}$. After that the sections were washed again by PBS for three times ( $5 \mathrm{~min}$ each), the same sections were then stained with DAPI $\left(4^{\prime}, 6\right.$-diamidino2-phenylindole) to recognize nuclei. The TUNEL positive cells were observed and counted under a fluorescence microscope (Olympus BX53, Japan).

\section{Subcellular Fractionation}

The subcellular proteins were fractionated using a membrane protein and a cytoplasmic protein extraction kit (Beyotime Biotechnology, Jiangsu, China) according to the manufacturer's instructions. Proteins (60 $\mu \mathrm{g} / \mathrm{sample})$ were separated by sodium dodecyl sulfatepolyacrylamide gel electrophoresis. $\mathrm{Na}, \mathrm{K}$-ATPase, and $\beta$ actin were employed as membrane or cytoplasmic loading controls.

\section{Western Blot Analysis}

Heart tissues were lysed using standard procedures, and protein concentration was determined using a BCA protein assay kit (Beyotime Biotechnology). Equal quantities of proteins were separated by SDS-PAGE, transferred onto a nitrocellulose membrane, and blocked using 5\% skim milk in TBS-Tween 20 (TBST). Membranes were incubated with specific primary antibodies overnight, followed by incubation with horseradish peroxidase-conjugated secondary antibody. The immunoreactive proteins were detected using ECL chemiluminescent reagent (Thermo Scientific, Pittsburgh, PA, USA) system on a luminescent image analyzer, Tanon 6600 (Tanon, Shanghai, China). Quantitation was performed using Image Pro Plus 6.0 software (Media Cybernetics, Rockville, MD, USA).

\section{RNA Extraction and Quantitative Real-Time PCR}

Total RNA was isolated using Trizol reagent following the manufacturer's instructions. The extracted RNA was reverse-transcribed to cDNA using iScript cDNA synthesis kit (Bio-Rad, Hercules, CA, USA). Quantitative analysis of mRNA was carried out using a SsoFast ${ }^{\text {TM }}$ EvaGreen ${ }^{\circledR}$ Supermix (Bio-Rad) with LightCycler 480 Real-Time PCR system (Roche Applied Science, Penzberg, Germany). The following primer sequences in Table 1 were used.

\section{Assessment of Oxidative Stress}

To analyze the redox status, total antioxidant capacity (T-AOC) in the myocardium was determined using commercial assay kits (Nanjing Jiancheng Bioengineering,

Table I Primers Used in Quantitative Real-Time PCR

\begin{tabular}{|l|l|}
\hline Primers & Sequence $\left(\mathbf{5}^{\prime}\right.$-3') \\
\hline NOX4 & $\begin{array}{l}\text { Forward: TGTTGGGCCTAGGATTGTGT } \\
\text { Reverse: CTTCTGTGATCCGCGAAGGT }\end{array}$ \\
\hline NOX2 & $\begin{array}{l}\text { Forward: TGCCAGTGTGTCGGAATCTC } \\
\text { Reverse: TGTGAATGGCCGTGTGAAGT }\end{array}$ \\
\hline$\beta$-actin & $\begin{array}{l}\text { Forward: GCAGGAGTACGATGAGTCCG } \\
\text { Reverse: ACGCAGCTCAGTAACAGTCC }\end{array}$ \\
\hline
\end{tabular}


Jiangsu, China). Oxidative stress in the form of intracellular reactive oxygen species (ROS) was tested via the 8-isoprostaglandin-F2 $\alpha$ (8-iso-PGF2 $\alpha$ ) assay with ELISA kit (Cayman Chemical, Ann Arbor, MI, USA). Meanwhile, DNA was extracted from myocardium and 8-hydroxy2 'deoxyguanosine (8-OHdG), a reliable marker of oxidative DNA damage was examined using an enzyme immunoassay (Cell Biolabs Inc, San Diego, CA, USA). All these experiments were performed strictly in accordance with the manufactures' instructions.

\section{Statistical Analysis}

All data are presented as the mean of at least three independent experiments. Each error bar represents the standard deviation. Statistical significance was determined using a one-way analysis of variance followed by the Student-Newman-Keuls comparison method to determine the differences between groups. Statistical analyses were performed using GraphPad Prism 8.0 software (GraphPad Software, San Diego, CA, USA). $p$ values $<0.05$ were considered to indicate statistically significant differences.

\section{Results}

\section{SMS Protects the Myocardium from} Diabetes-Induced Remodeling

Using electron microscopy analysis, Masson's trichrome and HE staining, we evaluated the myocardial ultrastructure, collagen deposition, and fibrosis of heart tissues. Transmission electron microscopy revealed that the nucleus showed an increase in heterochromatin lining the nuclear envelope and an increase in invaginations of the nuclear membrane in cardiomyocytes from diabetic rats; moreover, the fibrous region of mitochondria had an uneven, fragmented, and swollen appearance, causing them to lose their recognizable crest. These characteristics were significantly mitigated in the SMS-treated rats (Figure 1A).

Masson's trichrome staining was used to observe collagen fibrosis. As shown in Figure 1B, red cardiomyocytes were arranged neatly and tightly in normal rats, with a small amount of blue collagen fibers scattered in the intercellular space. The collagen tissue in the normal myocardium was organized, and the collagen fiber network among adjacent cells was intact with less collagen fiber content. In contrast, diabetic rats exhibited deposition of a large quantity of blue-stained, broken, and disordered collagen fibers around myocardial cells. However, the distribution of myocardial collagen fibers and interstitial fibrosis were remarkably reduced by SMS, with cardiomyocytes arranged in an orderly manner.

DCM characteristics were also observed in the leftventricular sections using hematoxylin and eosin staining (Figure 1C). Rats in the NC group had healthy cardiomyocytes, demonstrated as orderly arranged myocardial fibers, uniformly sized nuclei ranking in the central region of the cells, and clear stripes. However, in the DM group, the myocardial fibers were disordered, the nucleus sizes were different, and more vacuoles and myocardial fiber collapse were observed. Treatment with SMS protected heart tissues against the pathological changes due to DCM.

\section{SMS Treatment Attenuates Myocardial Apoptosis in DM Rats}

To evaluate the effect of SMS on cardiomyocyte apoptosis in DM rats, TUNEL staining was performed. As shown in Figure 2, the percentage of TUNEL-positive cells (stained red) in the DM group was higher than that in the $\mathrm{NC}$ group $(p<0.01)$, confirming increased apoptosis of the myocardium in the DM group. However, treatment with SMS significantly decreased the percentage of apoptotic myocardial cells (Figure 2; $p<0.01$ ). These results indicate that SMS could exert cardioprotective effects in DM by attenuating cardiomyocyte apoptosis.

\section{SMS Prevents Oxidative Stress in the Diabetic Myocardium}

Hyperglycemia-induced oxidative stress plays a critical role in the pathogenesis of DCM. Here, we quantified the total antioxidant capacity and levels of two reliable markers of oxidative injury in the myocardium. The total antioxidant capacity level in cardiac tissues in DM rats decreased significantly compared with that in the controls, which could be counteracted by SMS administration (Figure 3A). Moreover, the levels of both 8-isoPGF2 $\alpha$ and $8-\mathrm{OHdG}$ in myocardial tissues of DM rats were higher than those in normal hearts (Figure 3B and C), and treatment with SMS markedly attenuated the increase of these two molecules. As reported previously, 8-iso-PGF2 $\alpha$ and 8-OHdG represent the status of lipid peroxidation and DNA lesions, respectively, which are two major biochemical changes associated with oxidative damage. Therefore, our results suggest that SMS enhances antioxidant activity and protects the myocardium from oxidative injuries in DM. 


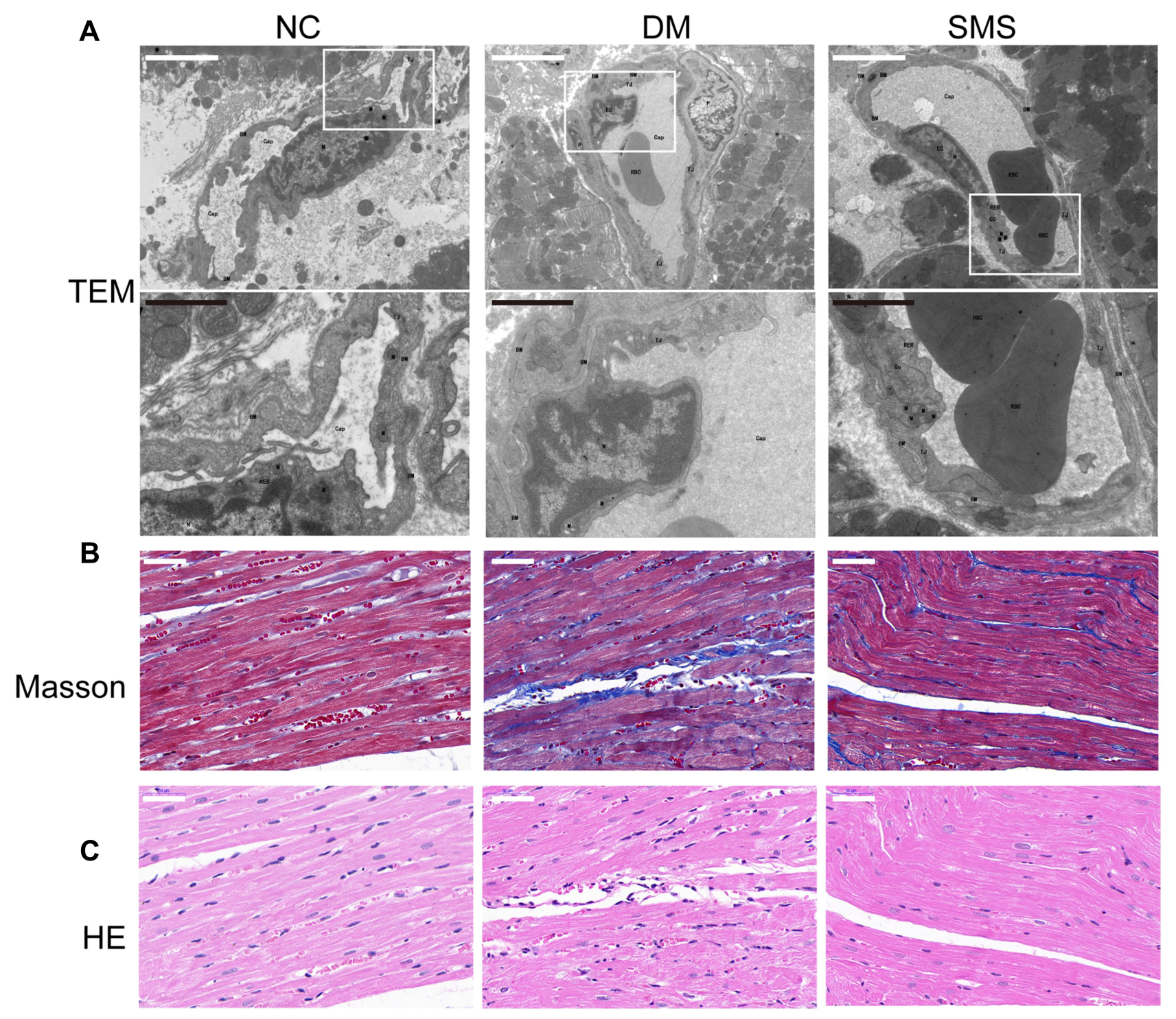

Figure I SMS protects ultrastructure integrity and attenuates cardiac fibrosis in diabetic rats. (A) Representative transmission electron micrographs of cardiac tissues (magnification = 15,000x). Scale bar (white) $50 \mu \mathrm{m}$; scale bar (black) $20 \mu \mathrm{m}$. (B) Representative images of myocardial tissue sections stained with Masson's trichrome solution (magnification $=400 \times$ ). Scale bar $50 \mu \mathrm{m}$. (C) Standard pictures of myocardial tissue sections stained with hematoxylin and eosin (magnification $=400 \times$ ). Scale bar $50 \mu \mathrm{m}$.

\section{SMS Blocks NOX Activation in DM Rats}

NOX is the main source of reactive oxygen species (ROS) in the myocardium. Therefore, we measured the protein and mRNA levels of NOX2 and NOX4 using Western blotting and real-time PCR analysis. The expression levels of NOX2 and NOX4 mRNA were significantly elevated in the myocardium of diabetic rats (Figure 4A and B). SMS treatment significantly downregulated the mRNA expression of NOX2 and NOX4. Similarly, the protein levels of NOX2 and NOX4 were evidently increased in the DM group compared with those in the control group (Figure 4C-F). After administration of SMS for 10 weeks, these changes were remarkably attenuated (Figure 4-F).

\section{SMS Inhibits NOX Translocation in DM Rats}

The translocation of cytosolic components (p47phox and p67phox) to the membrane is a prerequisite for NOX activation. To analyze NOX activity, we monitored the translocation to the cell membrane by quantifying the p47phox and p67phox contents in the cytoplasm and cytomembrane. As shown in Figure $5 \mathrm{~A}-\mathrm{C}$, under diabetic conditions, the level of p47phox in the cytomembrane was markedly higher, whereas it was significantly lower in the cytoplasm than in the normal myocardium. Treatment with SMS prevented the translocation of p47phox from the cytoplasm to the cardiac cell membrane. In parallel, diabetes induced an increase in the cytomembrane 


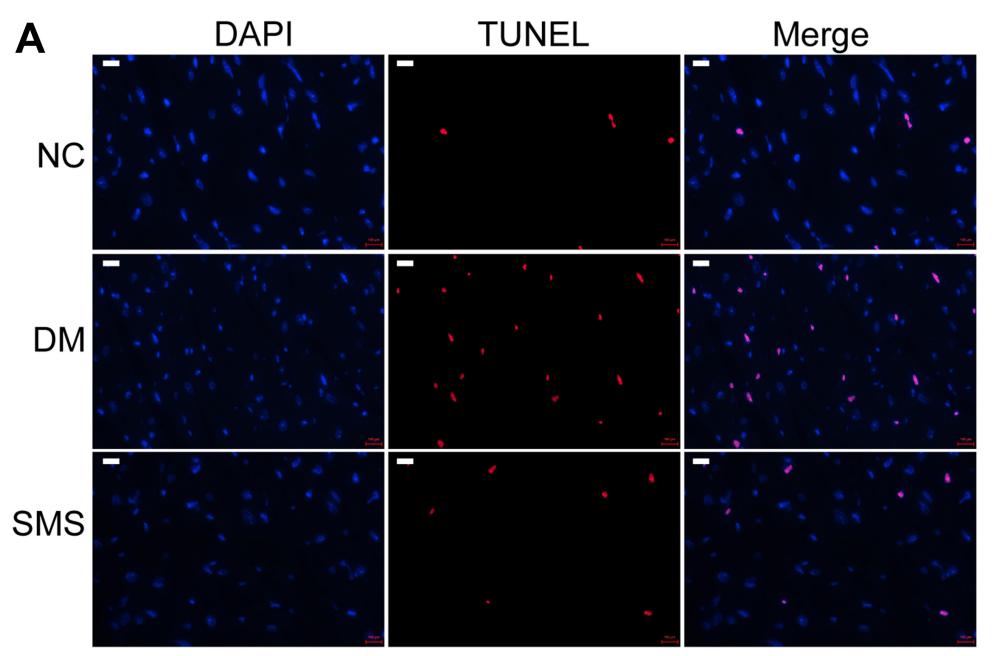

B

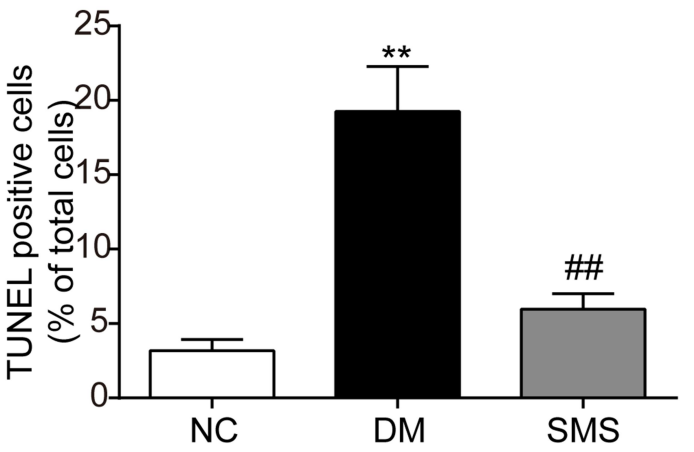

Figure 2 SMS protects cardiomyocytes from apoptosis induced by diabetes. $(\mathbf{A})$ Standard pictures of myocardial tissue sections stained with TUNEL (magnification $=630 \times$ ). Scale bar $100 \mu \mathrm{m}$. (B) Ratios of TUNEL-positive cells in different groups ( $\mathrm{n}=6$ rats per group). Results are presented as means \pm standard deviations. $* * p<0.0 \mathrm{I}$ vs the NC group and ${ }^{\# \#}<0.01$ vs the DM group.
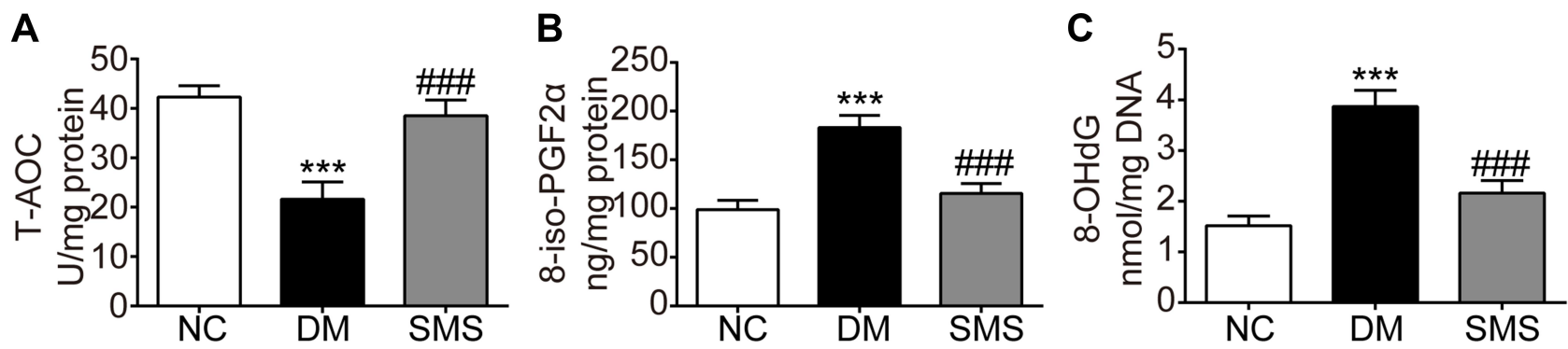

Figure 3 SMS prevents oxidative stress in the diabetic myocardium. Oxidative stress in the myocardial tissues was determined by measuring levels of (A) T-AOC, (B) 8-isoPGF2 $\alpha$, and (C) $8-O H d G$. Results are presented as means \pm standard deviation. ${ }^{* * *} p<0.001$ vs the NC group and ${ }^{\# \#} p<0.001$ vs the DM group ( $=6$ rats per group).

levels and a decrease in the cytoplasm levels of p67phox in the heart. The membrane translocation of p67phox was noticeably reduced in SMS-treated rats compared with that in DM rats (Figure 5D-F). These data indicate that in diabetic settings, NOX was activated and could be inhibited by SMS.

\section{SMS Promotes AMPK Activation in DM Rats}

Our data defined the inhibitory effects of SMS on NOX activation and oxidative injuries in the diabetic myocardium. Therefore, we tested whether SMS could stimulate AMPK, a critical signaling pathway that regulates oxidative stress and exerts protective effects against diabetes and a plethora of cardiovascular diseases. ${ }^{11}$ As illustrated in Figure 6A and B, diabetes attenuated the phosphorylation of AMPK $\alpha$ at Thr172, which positively correlates with its active status. SMS treatment restored AMPK $\alpha$ activity to nearly normal status. Our data suggest that SMS stimulates the AMPK pathway, which may reduce oxidative stress and provide cardio-protection.

\section{Discussion}

In the present study, we demonstrated that SMS is effective in attenuating diabetes-related cardiac remodeling, including collagen deposition, fibrosis, and apoptosis. Furthermore, the cardioprotective effects of SMS are partly mediated by AMPK activation, NOX suppression, and subsequent oxidative stress. Our findings suggest that SMS may be a potential candidate for the treatment of DCM.

Animal models of DCM have been established using various methods. Among these, low-dose STZ injection with a constant HFD mostly resembles the pathophysiology of type 2 DM. Reportedly, with continuous HFD intake, cardiac hypertrophy, fibrosis, and collagen fibers appeared after only 4 weeks of STZ injection. ${ }^{8,10}$ As expected, we detected fibrosis, apoptosis, and other pathological changes in the myocardium of rats treated with a long-term HFD and low-dose STZ. Furthermore, myocardial apoptosis is recognized as a key pathological feature of DCM. ${ }^{11}$ Myocardial apoptosis promotes the loss of contractile units and reparative fibrosis in the heart of 

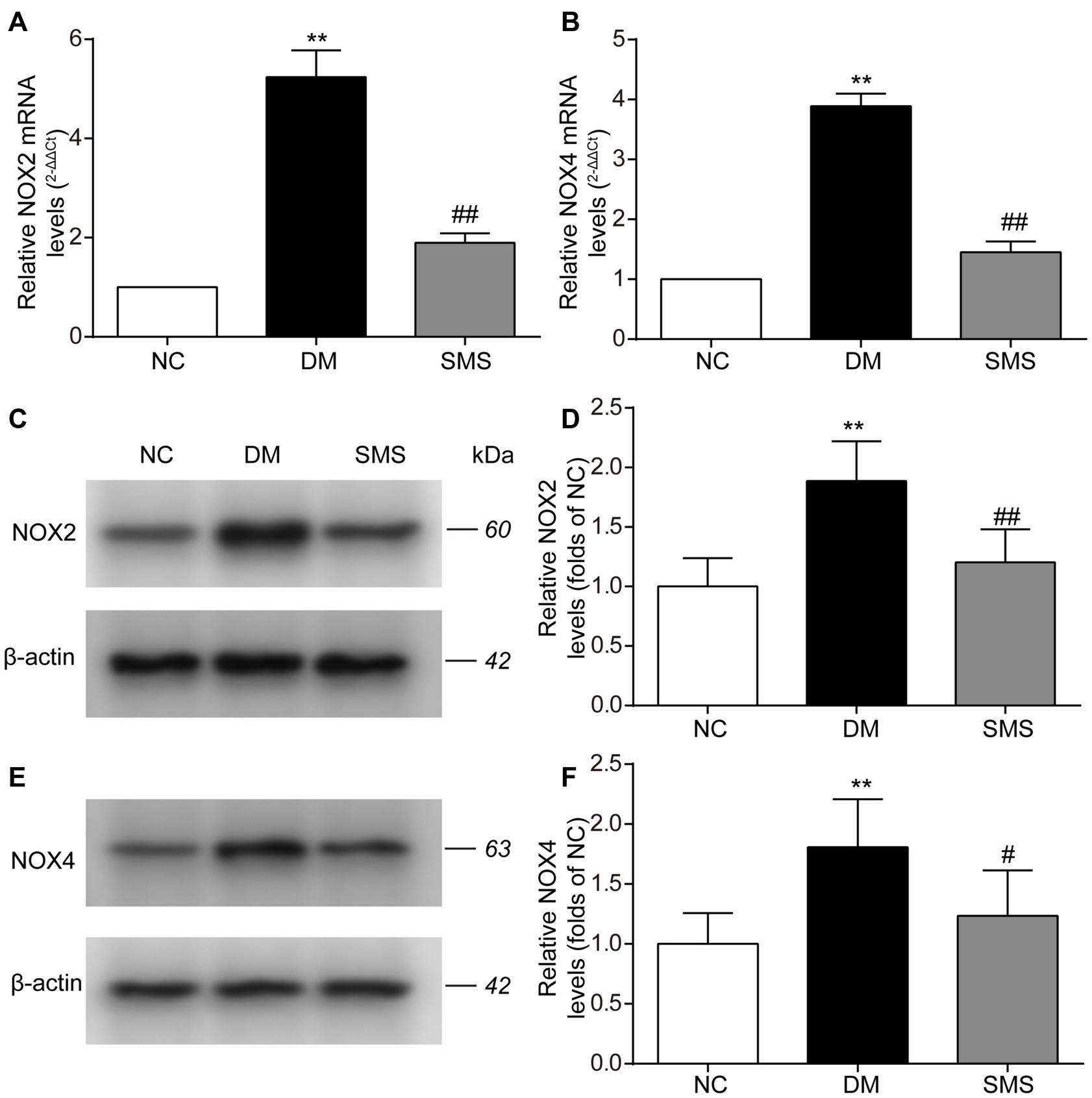

Figure 4 SMS blocks NOX activation in STZ-induced DM rats. (A and B) Real-time quantitative polymerase chain reaction and quantitative analysis of NOX2 and NOX4 mRNA levels in heart tissues. (C-F) Representative Western blot and quantitative analyses of NOX2 and NOX4 protein levels in heart tissues. $\beta$-actin was used as the internal control. Results are presented as means \pm standard deviations. ${ }^{* *} p<0.01$ vs the $N C$ group; ${ }^{*} p<0.05$ vs the DM group, and ${ }^{\# \#} p<0.01$ vs the DM group ( $n=6$ rats per group).

diabetic rats. ${ }^{12}$ Thus, cardiac apoptosis has been considered to be a therapeutic target for DCM. ${ }^{13}$ In this study, we observed apoptosis and fibrosis in the myocardium of diabetic rats, as indicated by swollen mitochondria, increased TUNEL-positive cardiomyocytes and deposition of collagen fibers. Although we did not test diastolic and systolic functions, apoptosis in combination with other structural abnormalities favored the induction of DCM. This result is consistent with that of a previous study. ${ }^{14}$
SMS has been clinically used in patients with coronary heart diseases, cardiogenic shock, and congestive heart failure for more than 1000 years in China. Recently, multiple studies have revealed the protective effects of SMS on the cardiovascular system. Using histological and morphological assessments, Zhao et al discovered that SMS prevents cardiac hypertrophy and ultrastructural injury in $\mathrm{db} / \mathrm{db}$ mice. ${ }^{7}$ In rats with myocardial ischemia-reperfusion injury, SMS could improve cell apoptosis. ${ }^{15} \mathrm{Ni}$ et al found that SMS inhibited 
A

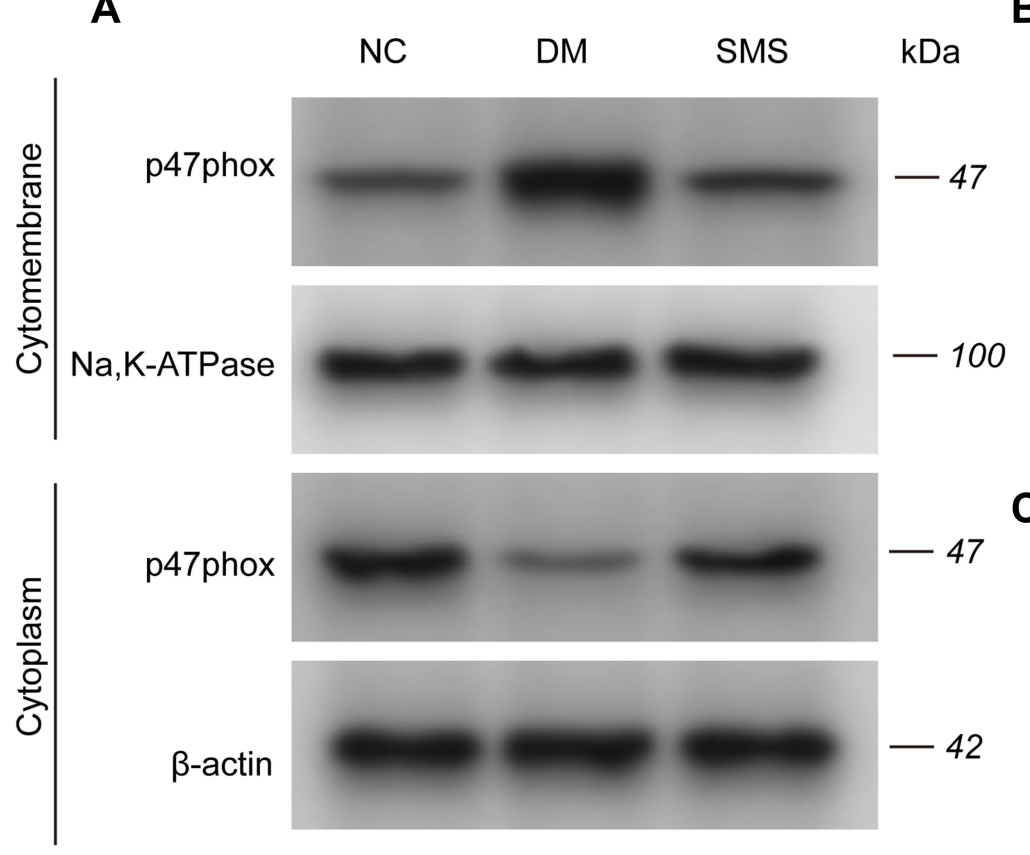

D

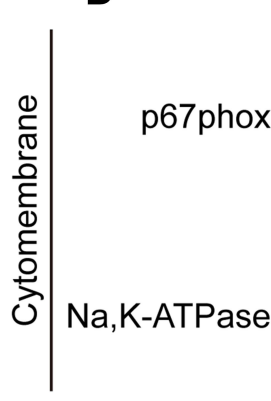

NC

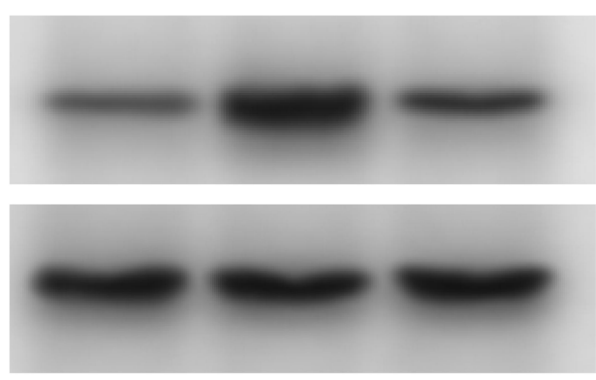

$\mid$\begin{tabular}{c|}
$\frac{1}{0}$ \\
$\frac{0}{0}$ \\
$\frac{0}{0}$ \\
$\frac{0}{0}$ \\
0
\end{tabular}

p67phox

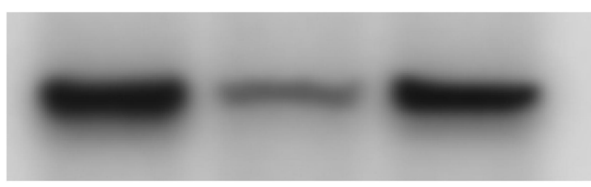

B
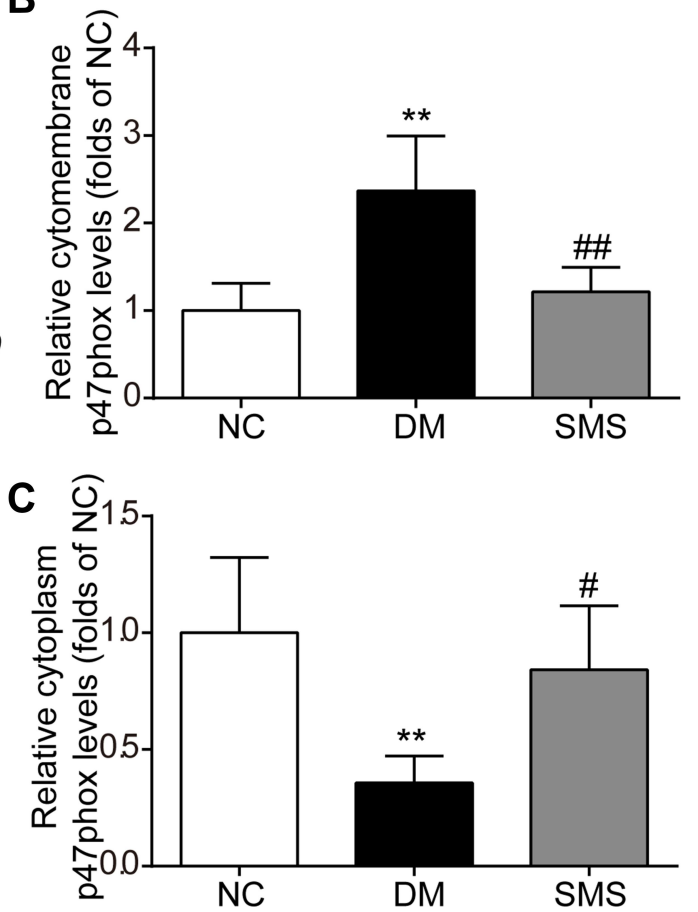

$\mathrm{kDa}$

E
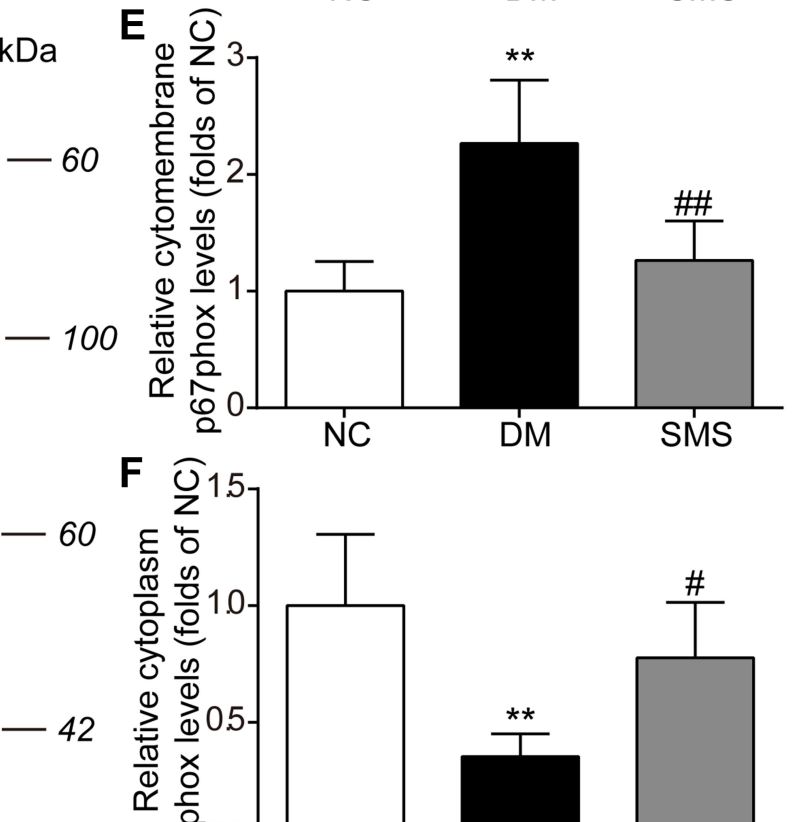

$F$
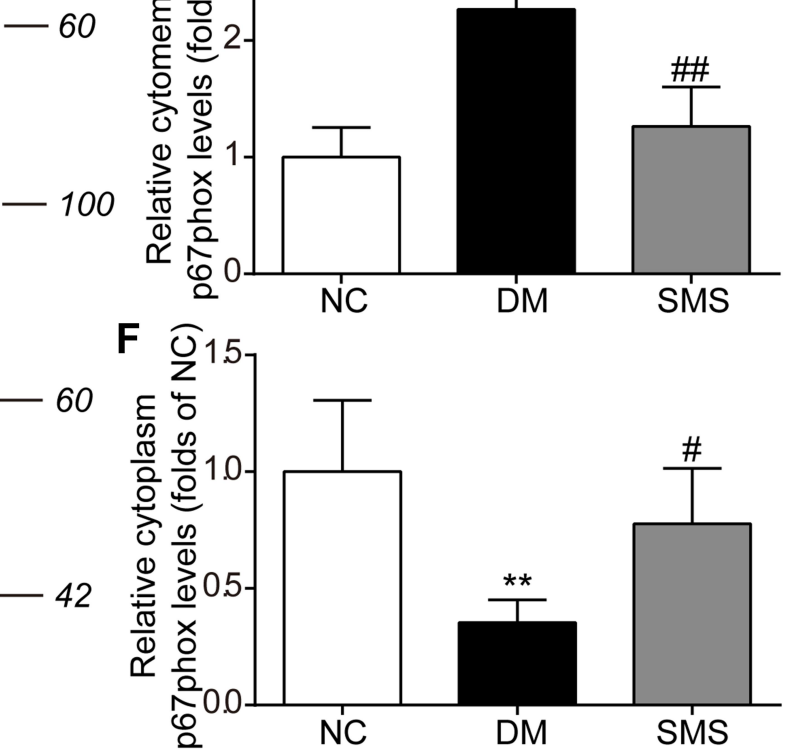

$-100$

Figure 5 SMS inhibits NOX translocation in DM rats. The subcellular levels of $p 47$ phox and p67phox protein in heart tissues from different groups were detected by Western blot, and representative bands are shown in (A and $\mathbf{D})$. The levels of cytomembrane and cytoplasm p47phox (B and $\mathbf{C})$, and cytomembrane and cytoplasm p67phox (E and F) were normalized to the NC group. The results are presented as means \pm standard deviations. ${ }^{* *} p<0.01$ vs the NC group, ${ }^{\#} p<0.05$ vs the DM group, and ${ }^{\#} p<0.01$ vs the DM group ( $n=6$ rats per group).

myocardial fibrosis, thus delaying the progression of cardiomyopathy in diabetic animals. ${ }^{6}$ Consistent with this result, our findings showed that 10 -week SMS treatment significantly decreased the percentage of apoptotic cardiomyocytes and improved most of the pathological changes in the myocardium of diabetic rats. This observation suggests that SMS ameliorates cardiac remodeling and exerts cardioprotective effects in diabetic rats.

In diabetes, hyperglycemia, hyperlipidemia, and other metabolic disorders lead to excessive reactive oxygen species (ROS) generation. Oxidative stress occurs when the production of ROS surpasses the degradation and is closely related to 


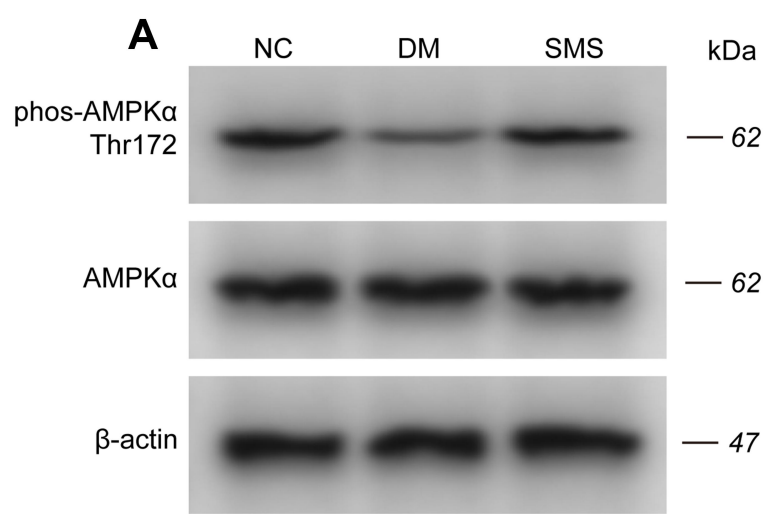

B

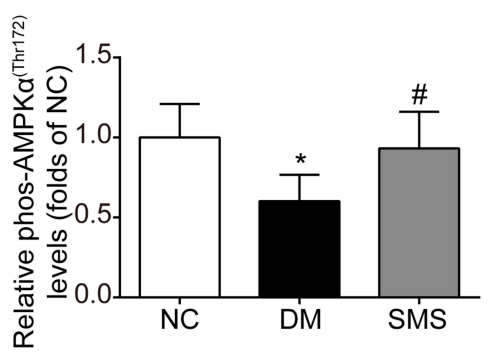

Figure 6 SMS restored AMPK activation in DM rats. The phosphorylation level of AMPK $\alpha$ in heart tissues from different groups was detected by Western blot assay. Representative bands were shown in $(\mathbf{A})$ Phosphorylation levels of AMPK $\alpha(\mathbf{B})$ were normalized to NC. The results were presented as mean \pm SD $(n=6)$. $* p<0.05$ vs the NC group, ${ }^{\#}<0.05$ vs the DM group.

various pathophysiological changes in DCM, including cardiac remodeling and dysfunction. ${ }^{16,17}$ ROS promotes myocardial fibrosis by inducing DNA damage, mitochondrial dysfunction, profibrotic factor generation, lipid and protein degeneration, and inflammatory response aggravation. ${ }^{18}$ Therefore, therapies that target intracellular ROS to relieve oxidative stress and related cardiac fibrosis may represent a potential strategy for the prevention of DCM. ${ }^{19}$ In this study, we analyzed the effects of SMS on oxidative stress and found that SMS enhances the total antioxidant capacity while decreasing the levels of cardiac 8-iso-PGF2 $\alpha$ and $8-\mathrm{OHdG}$, which are markers of oxidative modification in lipids and DNA. This observation suggests that the cardioprotective effects of SMS are correlated with redox signaling.

The mechanisms that are responsible for ROS generation in the diabetic myocardium include mitochondria, NOX, dysfunctional nitric oxide synthase (NOS) and xanthine oxidase (XO). ${ }^{5,20,21}$ Many of the systemic changes in diabetes are known activators of NOXs. High glucose and/or free fatty acids promote the recruitment of NOX catalytic subunits and activation of NOX. ${ }^{22,23}$ This activation results in the generation of superoxide, which consequently promotes mitochondrial ROS production in a positive feedback loop. ${ }^{24,25}$ Thus, NOXs are believed to be a major source of ROS in different organs and have been suggested as a therapeutic target for diabetic complications. $^{26}$ In the present study, we examined the expression and activity of NOX2 and NOX4, the major types of NOX in the myocardium. ${ }^{27}$ Our data showed that SMS reversed the elevation of cardiac NOX2 and NOX4 proteins while inhibiting the translocation of $\mathrm{p} 47 \mathrm{phox}$ and p67phox to the cytomembrane. Ginsenoside Rg1, one of the active ingredients of ginseng, was previously shown to downregulate NOX2 and reduce the production of ROS in $\mathrm{H}_{2} \mathrm{O}_{2}$-treated hippocampal neurons. ${ }^{28}$ Therefore, our results indicate that SMS may ameliorate DCM by reducing NOX activity and scavenging ROS derived from NOX.

AMPK, an energy sensor, is involved in glucose and fatty acid metabolism in mammalian cells. ${ }^{29}$ Recent evidences have shown that AMPK protects against oxidative stress in the heart under various pathological conditions. ${ }^{30}$ Specifically, AMPK has been reported to exert antioxidant effects in the cardiovascular system by inhibiting the expression and activity of NOX. ${ }^{31,32}$ Here, we found that SMS markedly increased AMPK $\alpha$ phosphorylation at Thr172 in the myocardium exposed to long-term high glucose. Our result was consistent with that of a study on $\mathrm{db} / \mathrm{db}$ mice that reported marked SMS enhancement of p-AMPK $\alpha$ protein levels in diabetic hearts. ${ }^{8}$

Taken together, using HFD- and STZ-induced diabetic rats, we demonstrated that SMS attenuated high glucose-induced myocardial injury by decreasing oxidative stress. The alleviation of redox reactions was associated with the inhibition of cardiac NOXs and activation of AMPK. Our results further confirmed the therapeutic potential of SMS in the treatment of diabetic cardiovascular complications. Intensive studies are required to elucidate the underlying mechanisms of SMS.

\section{Abbreviations}

DCM, diabetic cardiomyopathy; SMS, ShengMai-San; NOX, nicotinamide adenine dinucleotide phosphate oxidases; $\mathrm{NC}$, normal group; DM, diabetes mellitus; HE, haematoxylin eosin; TUNEL, terminal deoxynucleotidyl transferasemediated dUTP nick end labelling; T-AOC, total antioxidant capacity; 8-iso-PGF2 $\alpha$, 8-iso-prostaglandin-F2 $\alpha$; 8 -OhdG, 8-hydroxy-2'deoxyguanosine; $\mathrm{AMPK} \alpha, \quad$ adenosine 
monophosphate-activated protein kinase $\alpha$; qPCR, quantitative real-time polymerase chain reaction; AGEs, advanced glycation end products; RAAS, renin-angiotensin-aldosterone system; ER, endoplasmic reticulum; STZ, streptozotocin; HFD, high-fat diet; TEM, transmission electron microscopy; DAPI, 4',6-diamidino-2-phenylindole; BCA, bicinchoninic acid; ECL, enhanced chemiluminescence; ROS, reactive oxygen species; NOS, NO synthase; XO, xanthine oxidase.

\section{Acknowledgment}

We thanked the staff in Experimental Center, Shandong University of Traditional Chinese Medicine for the technical supports.

\section{Author Contributions}

All authors made substantial contributions to conception and design, acquisition of data, or analysis and interpretation of data; took part in drafting the article or revising it critically for important intellectual content; agreed to submit to the current journal; gave final approval of the version to be published; and agreed to be accountable for all aspects of the work.

\section{Funding}

This study was supported by the grants from the National Natural Science Foundation of China (grant number 81200592 and 81704038), the 56th general funded projects of China Postdoctoral Science Foundation (grant number 2014M561960), Shandong Medical and Health Science and Technology Development Plan Project (grant number 2015WS0143), and Natural Science Foundation of Shandong Province (grant number ZR2020QH329 and ZR2020MH387).

\section{Disclosure}

The authors have no conflicts of interest for this work.

\section{References}

1. Cho NH, Shaw JE, Karuranga S, et al. IDF diabetes atlas: global estimates of diabetes prevalence for 2017 and projections for 2045. Diabetes Res Clin Pract. 2018;138:271-281. doi:10.1016/j.diabres.2018.02.023

2. Gregg EW, Sattar N, Ali MK. The changing face of diabetes complications. Lancet Diabetes Endocrinol. 2016;4(6):537-547. doi:10.1016/S2213-8587(16)30010-9

3. Liu Q, Wang S, Cai L. Diabetic cardiomyopathy and its mechanisms: role of oxidative stress and damage. J Diabetes Investig. 2014;5 (6):623-634. doi:10.1111/jdi.12250

4. Zaveri MP, Perry JC, Schuetz TM, Memon MD, Faiz S, Cancarevic I. Diabetic cardiomyopathy as a clinical entity: is it a myth? Cureus. 2020;12(10):e11100.
5. Kaludercic N, Di Lisa F. Mitochondrial ROS formation in the pathogenesis of diabetic cardiomyopathy. Front Cardiovasc Med. 2020;7:12. doi:10.3389/fcvm.2020.00012

6. Ni Q, Wang J, Li EQ, et al. Study on the protective effect of shengmai san (see text) on the myocardium in the type 2 diabetic cardiomyopathy model rat. $J$ Tradit Chin Med. 2011;31(3):209-219. doi:10.1016/S0254-6272(11)60044-7

7. Zhao J, Cao TT, Tian J, et al. Shengmai san ameliorates myocardial dysfunction and fibrosis in diabetic $\mathrm{db} / \mathrm{db}$ mice. Evid Based Complement Alternat Med. 2016;2016:4621235. doi:10.1155/2016/ 4621235

8. Tian J, Tang W, Xu M, et al. Shengmai san alleviates diabetic cardiomyopathy through improvement of mitochondrial lipid metabolic disorder. Cell Physiol Biochem. 2018;50(5):1726-1739. doi:10.1159/000494791

9. Zhang SY, Yang KL, Long ZY, Li WQ, Huang HY. Use of a systematic pharmacological methodology to explore the mechanism of shengmai powder in treating diabetic cardiomyopathy. Med Sci Monit. 2020;26:e919029.

10. Jin L, Zhang J, Deng Z, et al. Mesenchymal stem cells ameliorate myocardial fibrosis in diabetic cardiomyopathy via the secretion of prostaglandin E2. Stem Cell Res Ther. 2020;11(1):122. doi:10.1186/ s13287-020-01633-7

11. Cai L, Kang YJ. Cell death and diabetic cardiomyopathy. Cardiovasc Toxicol. 2003;3(3):219-228. doi:10.1385/CT:3:3:219

12. Engel D, Peshock R, Armstong RC, Sivasubramanian N, Mann DL. Cardiac myocyte apoptosis provokes adverse cardiac remodeling in transgenic mice with targeted TNF overexpression. Am J Physiol Heart Circ Physiol. 2004;287(3):H1303-11. doi:10.1152/ ajpheart.00053.2004

13. Yao Q, Ke ZQ, Guo $S$, et al. Curcumin protects against diabetic cardiomyopathy by promoting autophagy and alleviating apoptosis. J Mol Cell Cardiol. 2018;124:26-34. doi:10.1016/j.yjmcc.201 8.10 .004

14. Yu W, Zha W, Guo S, Cheng H, Wu J, Liu C. Flos Puerariae extract prevents myocardial apoptosis via attenuation oxidative stress in streptozotocin-induced diabetic mice. PLoS One. 2014;9(5):e98044. doi:10.1371/journal.pone.0098044

15. Liu X, Tan W, Yang F, et al. Shengmai injection reduces apoptosis and enhances angiogenesis after myocardial ischaemia and reperfusion injury in rats. Biomed Pharmacother. 2018;104:629-636. doi:10.1016/j.biopha.2018.04.180

16. Yan B, Ren J, Zhang Q, et al. Antioxidative effects of natural products on diabetic cardiomyopathy. $J$ Diabetes Res. 2017;2017:2070178. doi:10.1155/2017/2070178

17. Lorenzo O, Ramirez E, Picatoste B, Egido J, Tunon J. Alteration of energy substrates and ROS production in diabetic cardiomyopathy. Mediators Inflamm. 2013;2013:461967. doi:10.1155/2013/461967

18. Tan Y, Zhang Z, Zheng C, Wintergerst KA, Keller BB, Cai L. Mechanisms of diabetic cardiomyopathy and potential therapeutic strategies: preclinical and clinical evidence. Nat Rev Cardiol. 2020;17(9):585-607.

19. Ma W, Guo W, Shang F, et al. Bakuchiol alleviates hyperglycemia-induced diabetic cardiomyopathy by reducing myocardial oxidative stress via activating the SIRT1/Nrf2 signaling pathway. Oxid Med Cell Longev. 2020;2020:3732718. doi:10.1155/ 2020/3732718

20. D'Oria R, Schipani R, Leonardini A, et al. The role of oxidative stress in cardiac disease: from physiological response to injury factor. Oxid Med Cell Longev. 2020;2020:5732956. doi:10.1155/2020/ 5732956

21. Koju N, Taleb A, Zhou J, et al. Pharmacological strategies to lower crosstalk between nicotinamide adenine dinucleotide phosphate (NADPH) oxidase and mitochondria. Biomed Pharmacother. 2019;111:1478-1498. doi:10.1016/j.biopha.2018.11.128 
22. Sedeek M, Montezano AC, Hebert RL, et al. Oxidative stress, Nox isoforms and complications of diabetes-potential targets for novel therapies. J Cardiovasc Transl Res. 2012;5(4):509-518. doi:10.1007/ s12265-012-9387-2

23. Brandes RP, Weissmann N, Schroder K. Nox family NADPH oxidases: molecular mechanisms of activation. Free Radic Biol Med. 2014;76:208-226. doi:10.1016/j.freeradbiomed.2014.07.046

24. Xiao S, Zang J, Pei Y, et al. Activation of mitochondrial orf355 gene expression by a nuclear-encoded DREB transcription factor causes cytoplasmic male sterility in maize. Mol Plant. 2020;13 (9):1270-1283. doi:10.1016/j.molp.2020.07.002

25. Hou Y, Ouyang X, Wan R, Cheng H, Mattson MP, Cheng A Mitochondrial superoxide production negatively regulates neural progenitor proliferation and cerebral cortical development. Stem Cells. 2012;30(11):2535-2547. doi:10.1002/stem.1213

26. Laddha AP, Kulkarni YA. NADPH oxidase: a membrane-bound enzyme and its inhibitors in diabetic complications. Eur J Pharmacol. 2020;881:173206. doi:10.1016/j.ejphar.2020.173206

27. Hansen SS, Aasum E, Hafstad AD. The role of NADPH oxidases in diabetic cardiomyopathy. Biochim Biophys Acta Mol Basis Dis. 2018;1864(5):1908-1913. doi:10.1016/j.bbadis.2017.07.025
28. Xu TZ, Shen XY, Sun LL, et al. Ginsenoside Rg1 protects against $\mathrm{H} 2 \mathrm{O} 2$ induced neuronal damage due to inhibition of the NLRP1 inflammasome signalling pathway in hippocampal neurons in vitro. Int J Mol Med. 2019;43(2):717-726. doi:10.3892/ijmm.2018.4005

29. Lv ZM, Liu Y, Zhang PJ, et al. The role of AMPKalpha in high-glucose-induced dysfunction of cultured rat mesangial cells. Ren Fail. 2012;34(5):616-621. doi:10.3109/0886022X.2012.668491

30. Li T, Mu N, Yin Y, Yu L, Ma H. Targeting AMP-activated protein kinase in aging-related cardiovascular diseases. Aging Dis. 2020;11 (4):967-977. doi:10.14336/AD.2019.0901

31. Song P, Zou MH. Regulation of NAD(P)H oxidases by AMPK in cardiovascular systems. Free Radic Biol Med. 2012;52 (9):1607-1619. doi:10.1016/j.freeradbiomed.2012.01.025

32. Ceolotto G, Gallo A, Papparella I, et al. Rosiglitazone reduces glucose-induced oxidative stress mediated by NAD $(\mathrm{P}) \mathrm{H}$ oxidase via AMPK-dependent mechanism. Arterioscler Thromb Vasc Biol. 2007;27(12):2627-2633. doi:10.1161/ATVBAHA.107.155762

Diabetes, Metabolic Syndrome and Obesity: Targets and Therapy

Dovepress

\section{Publish your work in this journal}

Diabetes, Metabolic Syndrome and Obesity: Targets and Therapy is an international, peer-reviewed open-access journal committed to the rapid publication of the latest laboratory and clinical findings in the fields of diabetes, metabolic syndrome and obesity research. Original research, review, case reports, hypothesis formation, expert opinion and commentaries are all considered for publication. The manuscript management system is completely online and includes a very quick and fair peer-review system, which is all easy to use. Visit http://www.dovepress.com/testimonials.php to read real quotes from published authors.

Submit your manuscript here: https://www.dovepress.com/diabetes-metabolic-syndrome-and-obesity-targets-and-therapy-journal 